\title{
Boosting platelet inhibition in poor responder to aspirin and clopidogrel undergoing percutaneous coronary intervention: role of tirofiban
}

This article was published in the following Dove Press journal:

Journal of Blood Medicine

8 May 2010

Number of times this article has been viewed

\author{
Gianluca Campo' \\ Luca Fileti' \\ Marco Valgimigli' \\ Jlenia Marchesini ${ }^{\prime}$ \\ Antonella Scalone' \\ Roberto Ferrari ${ }^{1,2}$ \\ 'Cardiovascular Institute, Azienda \\ Ospedaliera Universitaria S Anna, \\ Ferrara, Italy; ${ }^{2}$ Cardiovascular \\ Research Centre, Salvatore Maugeri \\ Foundation, IRCCS Gussago (BS), Italy
}

Correspondence: Gianluca Campo Cardiovascular Institute, Azienda Ospedaliera Universitaria

S Anna, Ferrara, Italy

Tel +390532202143

Fax +390532241885

Email cmpglc@unife.it
Abstract: Nowadays, aspirin (acetylsalicylic acid) and clopidogrel form the cornerstone in prevention of cardiovascular events and their clinical effectiveness has been well established. The thienopyridine clopidogrel is a prodrug that, after hepatic metabolization, strongly inhibits adenosine diphosphate-induced platelet aggregation. Aspirin is a non-steroidal anti-inflammatory drug that exerts its anti-platelet action through the irreversible acetylation of platelet cyclooxygenase (COX)-1, blocking thromboxane A2 production. However, despite dual-antiplatelet therapy, some patients still develop recurrent cardiovascular ischemic events. Many studies have clearly showed that a marked variability exists in the responsiveness to aspirin and clopidogrel, being the poor responder patients at higher risk of short (peri-procedural) and long-term ischemic complications. In particular, these patients showed a major recurrence of myocardial infarction and, after stent implantation, of stent thrombosis. The mechanisms of aspirin and clopidogrel poor response are numerous and not fully elucidated, and are likely multifactorial (eg, genetic polymorphisms, elevated baseline platelet reactivity, drug interaction). How to improve the short- and long-term outcome of these patients is currently unknown. Recently published and ongoing clinical trials are evaluating different strategies for the acute and chronic treatments (eg, reload of clopidogrel, double clopidogrel maintenance dose, switching to prasugrel). In this paper, we reviewed all available evidence on aspirin and clopidogrel resistance and focused our attention on tirofiban, a glycoprotein IIb/IIIa inhibitor that may be used to obtain a better platelet inhibition in poor responder patients during the acute phase and in particular during percutaneous coronary intervention.

Keywords: aspirin, clopidogrel, tirofiban, resistance, VerifyNow, Multiplate Analyzer

\section{Introduction}

Cardiovascular diseases are the most common cause of mortality and morbidity in western countries in the 21 st century. Because aggregation of platelets highly contributes to the development of cardiovascular events, inhibition of this process plays an important role in the prevention of cardiovascular disease. Nowadays, aspirin (acetylsalicylic acid) and clopidogrel form the cornerstone in secondary prevention of cardiovascular events. The clinical effectiveness of aspirin on the prevention of cardiovascular events has been well established. The Antithrombotic Trialists' Collaboration has documented in their most recent meta-analysis of 287 randomized trials, incorporating more than 200000 patients, a $22 \%$ reduction of death and serious ischemic vascular events by antiplatelet therapy with aspirin compared with placebo. ${ }^{1}$ 
Similarly, many randomized clinical trials (eg, CAPRIE, ${ }^{2}$ CURE, ${ }^{3}$ PCI-CURE, ${ }^{4}$ CREDO $^{5}$ ) have shown the clinical effectiveness and utility of long-term clopidogrel treatment (alone or in association with aspirin) in reducing the combined risk of ischemic stroke, myocardial infarction (MI), and vascular death. However, not all patients profit to the same extent, which could be explained by a variety of pharmacodynamic, pharmacokinetic, and biochemical features. Addressed biochemically as persistent platelet reactivity in vitro despite use of aspirin and/or clopidogrel, this phenomenon is called 'resistance' or 'poor responsiveness' to oral antiplatelet agents (OAA; aspirin and clopidogrel), although a uniform definition is lacking.

In this paper, we reviewed all available evidence on aspirin and clopidogrel resistance and focused our attention on tirofiban, a glycoprotein IIb/IIIa inhibitor, which may be used to obtain a better platelet inhibition in poor responder patients during the acute phase and so overcome the higher risk of ischemic complications of these patients.

\section{Poor response to clopidogrel}

The thienopyridine clopidogrel is a prodrug that needs to be metabolized to an active compound that is a potent inhibitor of ADP induced platelet aggregation. ADP is a platelet activator that is secreted from platelets upon activation and is released from damaged vessels and red blood cells. Three ADP receptors have been described on platelets. P2X1 (a calcium channel), P2Y1 (coupled to Gq that mobilize intracellular calcium) and P2Y12, which leads to inhibition of adenylate cyclase via Gi-protein. The P2Y12 receptor has been identified as a target of clopidogrel (Table 1). More specifically, clopidogrel is oxidized in a cytochrome P450 (CYP) mono-oxygenase dependent way to 2-oxo-clopidogrel, an intermediate metabolite that is further hydrolyzed to the active thiol metabolite of clopidogrel. The active metabolite irreversibly binds to the $\mathrm{P} 2 \mathrm{Y} 12$ receptor (Figure 1). Although CYP3A4 is not the only cytochrome P450 isoenzyme involved in the metabolism of clopidogrel, it is quantitatively the most important one. The major circulating metabolite of clopidogrel is a carboxylic acid derivate that completely lacks of anti-aggregation activity. There is growing evidence suggesting that the response to clopidogrel shows an important interindividual variability ${ }^{6}$ and that may be influenced by pharmacokinetic variables such as intestinal absorption and metabolic activation in the liver, both of which are affected by genetic polymorphisms. ${ }^{7}$ In particular, previous studies clearly showed that a valuable group of patients has lower levels of clopidogrel active metabolite, diminished platelet inhibition, and higher rates of adverse cardiovascular events. ${ }^{8}$ Nowadays different assays are available to evaluate the clopidogrel induced platelet inhibition (clopidogrel response). The most used are light transmission aggregometry, VerifyNow, Multiplate Analyzer, and VASP index. Independently to the assay and the cut-off

Table I Principal characteristics of clopidogrel, aspirin and tirofiban

\begin{tabular}{|c|c|c|c|}
\hline & Clopidogrel & Aspirin & Tirofiban \\
\hline \multicolumn{4}{|l|}{ Characteristics } \\
\hline Compound & $\begin{array}{l}\text { Thienopyridine } \\
\text { (second generation) }\end{array}$ & $\begin{array}{l}\text { Non-steroidal } \\
\text { anti-inflammatory drug }\end{array}$ & Peptidomimetic nonpeptide \\
\hline Mechanism of action & $\begin{array}{l}\text { Irreversible and selective } \\
\text { antagonism of adenosine } \\
\text { diphosphate receptor, } \\
\text { subtype } \mathrm{P} 2 \mathrm{Y} / 2\end{array}$ & $\begin{array}{l}\text { Irreversible acetylation of } \\
\text { platelet cyclooxygenase } \\
(\text { COX)-I }\end{array}$ & $\begin{array}{l}\text { Competitive inhibitor of GP Ilb/IIla } \\
\text { platelet receptor }\end{array}$ \\
\hline Way of administration & per os & per os & intravenous \\
\hline Reversibility of effects & $5-8$ days & $8-10$ days & $<4$ hours \\
\hline Platelet bound half life & 4-5 days & 10 days & $\mathrm{IIs}$ \\
\hline Plasma half life & 6 hours & $15-20 \mathrm{~min}$ & $1.8 \mathrm{~h}$ \\
\hline Dose & $\begin{array}{l}\text { Loading dose: } \\
300 \text { or } 600 \mathrm{mg} \text { ( } 600 \mathrm{mg} \text { preferably) } \\
\text { Maintenace dose: } \\
75 \mathrm{mg} / \text { die }\end{array}$ & $\begin{array}{l}\text { Loading dose: } \\
\text { 150-325 mg } \\
\text { Maintenace dose: } \\
75-160 \mathrm{mg} / \text { die }\end{array}$ & $\begin{array}{l}\text { PRISM-PLUS dose (upstream therapy): } \\
0.4 \mu \mathrm{g} / \mathrm{kg} / \mathrm{min} \text { for } 30 \text { minutes + infusion } \\
0.1 \mu \mathrm{g} / \mathrm{kg} / \mathrm{min} \\
\text { Restore dose: } \\
10 \mu \mathrm{g} / \mathrm{kg} \text { bolus + infusion } 0.15 \mu \mathrm{g} / \mathrm{kg} / \mathrm{min} \\
\text { Single high dose bolus (downstream } \\
\text { therapy): } \\
25 \mu \mathrm{g} / \mathrm{kg} \text { bolus + infusion } 0.15 \mu \mathrm{g} / \mathrm{kg} / \mathrm{min}\end{array}$ \\
\hline Clearance & Renal $50 \%$, fecal $50 \%$ & Renal & Renal $(60 \%-70 \%)$, biliar $(20 \%-30 \%)$ \\
\hline
\end{tabular}




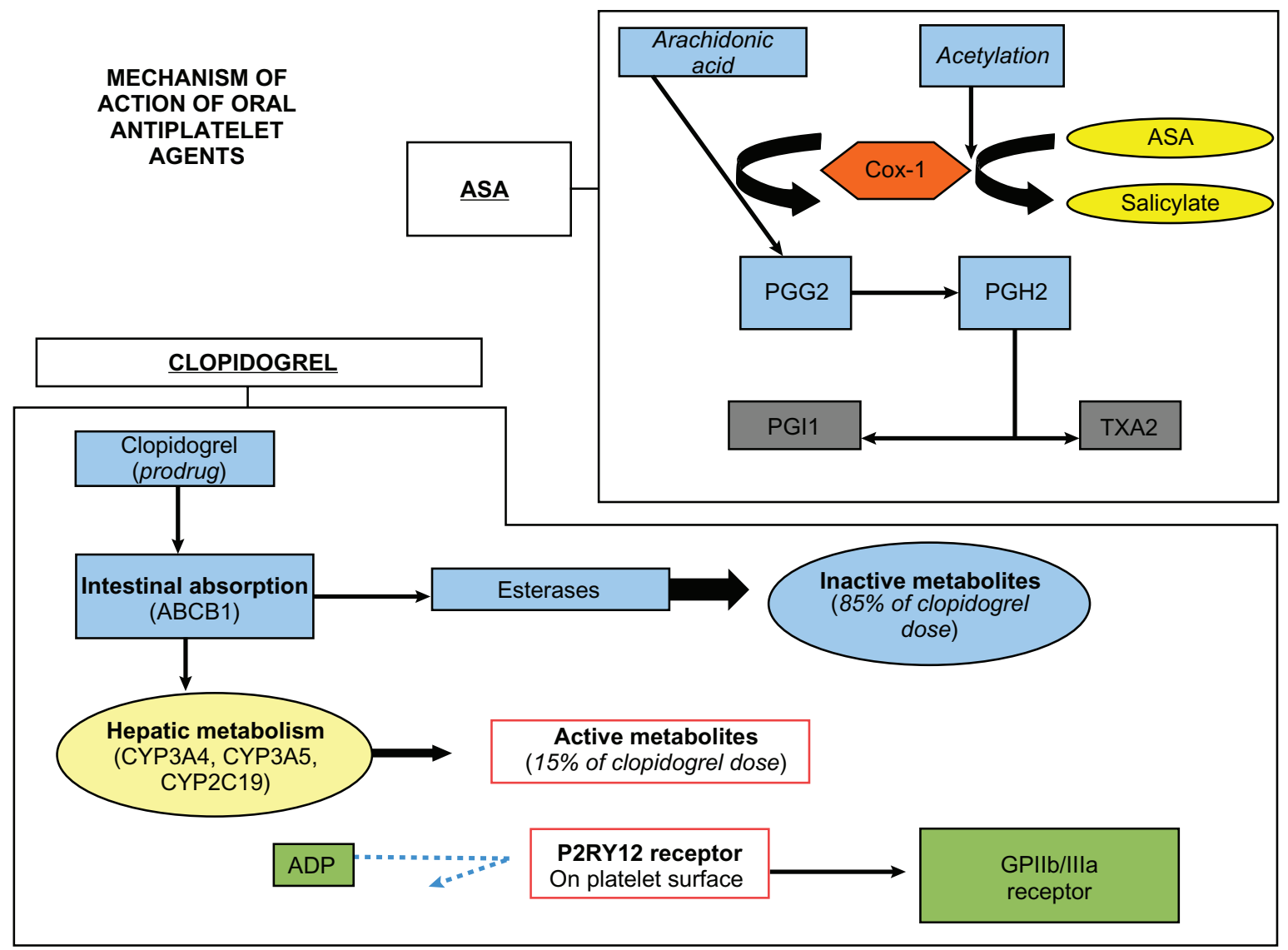

Figure I Mechanisms of action of aspirin and clopidogrel.

Abbreviations: ASA, aspirin; Cox, cyclo-oxygenase; PG, prostaglandin;Tx, thromboxane; ADP, adenosine diphosphate.

used, a strong association between clopidogrel response and adverse events has been observed. For example, using light transmission aggregometry, in the CREST (Clopidogrel Effect on Platelet Reactivity in Patients With Stent Thrombosis) study a relationship between high post-treatment platelet reactivity, incomplete P2Y12 receptor inhibition and sub acute stent thrombosis has been found, ${ }^{9}$ whereas in the EXCELSIOR study (Impact of Extent of ClopidogrelInduced Platelet Inhibition During Elective Stent Implantation on Clinical Event Rate) relatively low-risk patients undergoing percutaneous coronary intervention (PCI) at least 2 hours after $600 \mathrm{mg}$ loading dose of clopidogrel had higher risk of death, reinfarction, and stent thrombosis at 30 days. ${ }^{10}$ Marcucci et al showed that on-treatment residual platelet reactivity, measured by the point-of-care assay VerifyNow $\mathrm{P} 2 \mathrm{Y} 12$, is able to detect acute coronary syndrome patients at risk of 12-month cardiovascular death and nonfatal MI, founding, as optimal cutoff, the value of 240 P2Y12 reaction units. ${ }^{11}$ Using the same instrument and the same cutoff, Patti et al demonstrated that patients with high platelet reactivity during clopidogrel treatment (after $600 \mathrm{mg}$ loading dose) had higher risk of peri-procedural MI. ${ }^{12}$ Similarly in the study leaded by Sibbing et al using Multiplate Analyzer, has been found that clopidogrel poor responders, as compared to patients with a normal response, had a significantly higher risk of definite stent thrombosis $(2.2 \%$ vs $0.2 \% ; P<0.01)$ and mortality $(1.2 \%$ vs $0.4 \% ; P=0.07)$ within 30 days. ${ }^{13}$

\section{Poor response to aspirin}

Aspirin is a non steroidal anti-inflammatory drug that is quickly absorbed by gastrointestinal mucosa, and has a hepatic metabolism (Table 1 and Figure 1). Aspirin, or acetylsalicylic acid, exerts its anti-platelet action through the irreversible acetylation of platelet cyclooxygenase (COX)-1 at serine residue 530 (Figure 1). This enzyme is responsible for conversion of arachidonic acid to the various eicosanoids, such as leukotrienes, prostaglandins, thromboxane $\mathrm{A}_{2}\left(\mathrm{TXA}_{2}\right)$, and prostacyclin, or prostaglandin $\mathrm{I}_{2}\left(\mathrm{PGI}_{2}\right)$. Thromboxane $\mathrm{A}_{2}$ is a potent vasoconstrictor and platelet agonist found in the $\alpha$-granules of the platelet, which are released during platelet activation. Production of TXA ${ }_{2}$ has been completely inhibited by doses of aspirin as low as $100 \mathrm{mg}$. Despite several studies 
published on 'aspirin resistance', its definition, diagnosis, prevalence, causes, and clinical consequences are still uncertain. ${ }^{14}$ Direct comparison of different laboratory methods to detect aspirin resistance usually showed very weak or no correlation, indicating that they are sensitive to different parameters. In addition, the calculated prevalence of aspirin resistance in 11 studies that used different functional assays of platelet function, varied between 5.5\% and 61\%. ${ }^{15}$ Studies that measured TxB2 levels in aspirin-treated patients reported a prevalence of aspirin resistance that ranged between $1 \%$ and $1.7 \%{ }^{16}$ Therefore, aspirin resistance, when tested with appropriate, specific tests, appears to be extremely rare, and, in most instances, due to under-dosing or non-compliance. Nevertheless, several authors have found a good association between aspirin poor response and adverse events. For example, Eikelboom et al showed that suboptimal reduction of urinary 11-dehydro TxB2 levels during aspirin treatment is associated with heightened risk of future myocardial infarction and cardiovascular death. ${ }^{17}$ Similarly Gum et al found, in a cohort of 326 patients, 17 aspirin poor responders and 4 of these $(24 \%)$ experienced death, MI, or cerebrovascular accident, as compared with 30 of $309(10 \%)$ patients who were aspirin full responders $(P=0.03) .{ }^{18}$ Aspirin poor response emerged also at multivariable analyses as independent predictor. Finally, Chen et al showed that, in patients undergoing PCI, aspirin poor response, as evaluated by VerifyNow instrument, is associated with an higher incidence of cardiovascular death, myocardial infarction, stroke, and transient ischemic attack, in particular due to higher occurrence of peri-procedural MI. ${ }^{19}$

\section{Mechanism of antiplatelet resistance or variable response to oral antiplatelet agents}

The mechanisms of aspirin and clopidogrel poor response are numerous and not fully elucidated, and are likely to be multifactorial (Table 2). Below we have reported and discussed the most important.

\section{Genetic polymorphisms}

Previous studies have clearly identified different gene polymorphisms modulating clopidogrel absorption (eg, ABCB117), metabolic activation (eg, CYP3A519 and CYP2C19) and biologic activity (eg, P2RY1214 and ITGB311). ${ }^{7,8}$ Specifically, common polymorphisms in the CYP2C19 gene, seen in approximately $30 \%$ of whites, $40 \%$ of blacks, and more than $55 \%$ of east Asians, significantly diminish both the pharmacokinetic and pharmacodynamic responses to clopidogrel by approximately one quarter to one third. In particular, CYP2C19*2 is the most frequent variant allele (95\%) among the reduced-function group. This loss-of-function variant encodes a cryptic splice variant that leads to no enzymatic activity. Among clopidogrel-treated subjects in TRITON-TIMI 38, carriers of a reduced-function CYP2C19 allele had a relative increase of 53\% in the composite primary efficacy outcome of risk of death from cardiovascular causes, myocardial infarction, or stroke, as compared with non carriers $(12.1 \%$ vs $8.0 \%$; hazard ratio for carriers, $1.53 ; 95 \% \mathrm{CI}, 1.07$ to $2.19 ; P=0.01)$, and an

Table 2 Mechanisms of clopidogrel and aspirin poor response

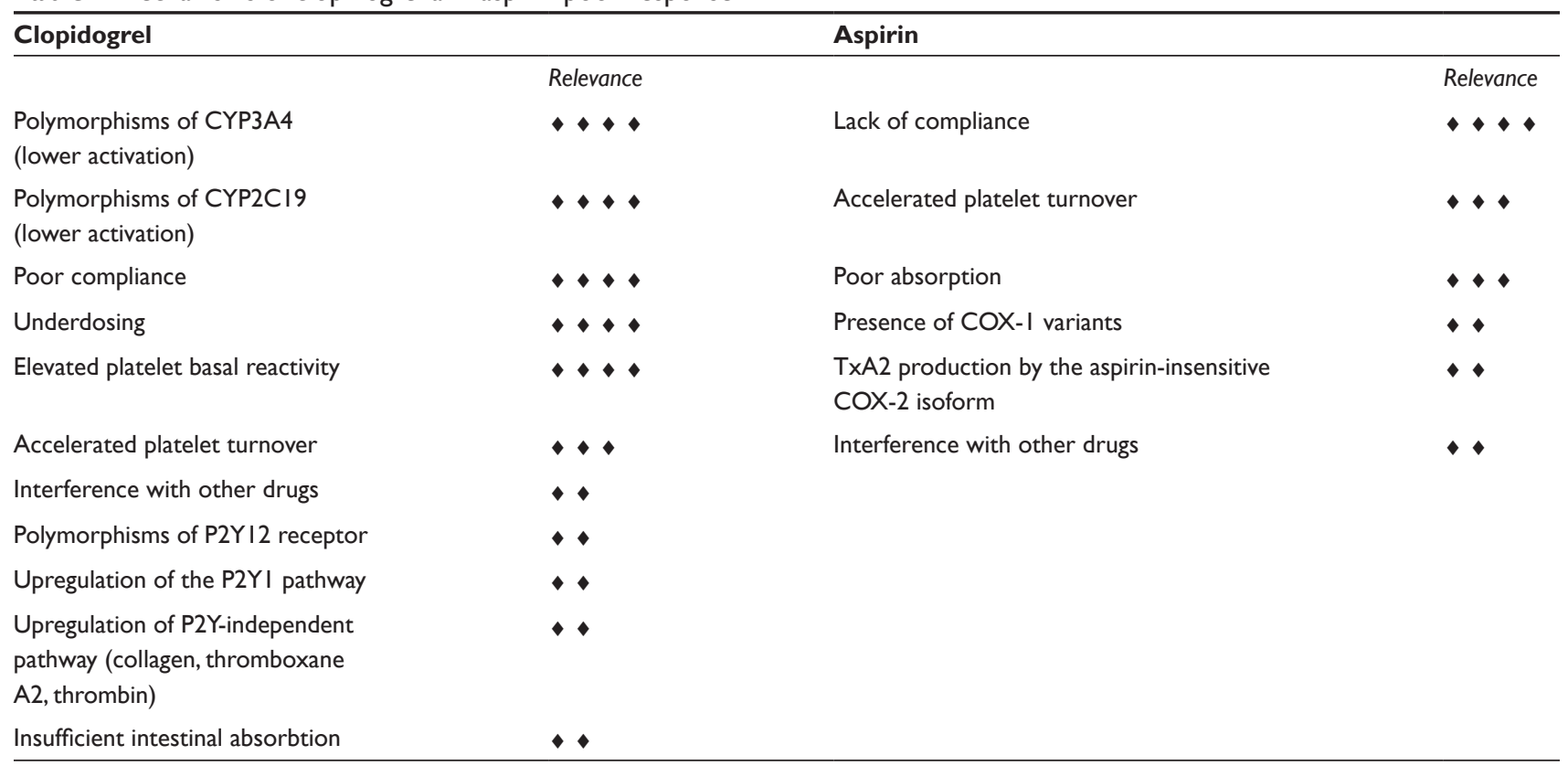


increase by a factor of 3 in the risk of stent thrombosis (2.6\% vs $0.8 \%$; hazard ratio, $3.09 ; 95 \%$ CI, 1.19 to 8.00 ; $P=0.02){ }^{8}$

\section{Interferences with other drugs}

Attention has been placed on a potential interaction observed between clopidogrel and the widely used proton pump inhibitors (PPIs). The CYP2C19 isoform is the key enzyme in the metabolism of many of the PPIs, which are also inhibitors of the CYP2C19 isoenzyme in varying degrees. In addition to metabolic inconsistencies, variability of intestinal absorption is also an important determinant of the wide response variability to clopidogrel. PPIs are substrates and inhibitors of the intestinal efflux transporter P-glycoprotein, a key factor for intestinal absorption of clopidogrel. Nevertheless, in a large population of patients with acute coronary syndrome undergoing percutaneous coronary intervention, the use of a PPI was not independently associated with increased risk of adverse clinical outcomes for patients treated with either clopidogrel or the novel thienopyridine prasugrel. ${ }^{20}$ As a contrary, an important competition of aspirin with other non steroidal anti-inflammatories (NSAIDs), such as ibuprofen, has been clearly reported. Ibiprofen can prevent aspirin access at Ser530 of COX-1 and, as a consequence, its irreversible acetylation and inactivation of the enzyme.

\section{Elevated baseline platelet reactivity}

In vitro data suggest that diabetes mellitus and acute coronary syndromes (ACS) may be associated with increased platelet reactivity. ${ }^{21,22}$ Many studies showed that patients with diabetes or presenting with ACS have larger platelets, an increased number of GP IIb/IIIa receptors on each platelet, and an increased population of activated circulating platelets, expressing, among other substances, P-selectin and thrombospondin. These adhesion molecules mediate platelet-leukocyte interactions and therefore are potential triggers of inflammatory response and thrombosis. In this specific subset we observed higher baseline platelet reactivity and, as consequence, both aspirin and clopidogrel effects are lower, and more time and higher dose are necessaries to obtain a significant platelet inhibition. ${ }^{21-23}$

\section{Accelerated platelet turnover}

An accelerated platelet turnover could be involved in the poor response to aspirin. It may introduce newly formed, non-aspirinated platelets into the blood stream, which are able to form TxA2. ${ }^{23}$

\section{Tirofiban}

The glycoprotein (GP) IIb/IIIa inhibitors are potent antagonists of platelet aggregation that are approved to prevent thrombotic complications of PCI and as medical treatment of patients with acute coronary syndromes (ACS). Tirofiban is a small synthetic nonpeptide, competitive GPIIb/IIIa inhibitor with high specificity and affinity for GPIIb/IIIa receptors conferred by a tyrosine analog structurally similar to the RGD (arginine-glycine-aspartic acid)-loop of the GPIIb/IIIa receptor. ${ }^{24}$ Tirofiban is administered as an intravenous infusion and approximately $35 \%$ is unbound in the circulation with predominant renal clearance $(65 \%)$, and it can be hemodialyzed (Table 1). Renal function may influence the excretion of tirofiban, but concurrent disease or other drugs generally used in patients with ischemia seem not to do so.

\section{Clinical efficacy of tirofiban}

Tirofiban has been tested both in patients with ACS, administered soon after hospital admission as part of the medical therapy, and in high-risk patients, including, but not limited to, those presenting with acute coronary disorders, administered immediately before PCI. In the PRISM (Platelet Receptor Inhibition for ischemic Syndrome Management) study, a total of 3231 patients presenting with ischemic symptoms of unstable angina were randomized to tirofiban or unfractioned heparin (UFH). ${ }^{25}$ In this trial the incidence of the primary composite endpoint (death, myocardial infarction, or refractory ischemia) at 48 hours was 32\% lower in the group that received tirofiban $(P=0.01)$ and, at 30 days, the mortality was $2.3 \%$ in the tirofiban arm and $3.6 \%$ in the heparin group $(P=0.02)$. Major bleeding occurred in $0.4 \%$ of patients in both groups. ${ }^{25}$ In the PRISM-PLUS trial a total of 1915 patients with high-risk non ST-segment elevation ACS were enrolled to evaluate tirofiban plus adjusted dose heparin, or adjusted dose heparin alone. ${ }^{26}$ The frequency of the composite primary endpoint at 7 days was lower among the patients who received tirofiban, in particular there was a $47 \%$ decrease in the risk of MI $(P=0.006)$ and a $30 \%$ decrease in the risk of refractory ischemia $(P=0.02)$ as compared with the risk in the heparinonly group. The risk of the composite endpoint of death or MI was reduced by $43 \%(P=0.006) .{ }^{26}$ Similarly, the RESTORE trial found a reduction in the incidence of composite endpoint (death, MI, coronary artery bypass graft, target vessel revascularization) at $2(P=0.005)$ and 7 days $(P=0.02)$ in the tirofiban group and a non statistically significant reduction of the endpoints at 30 days (from $12.2 \%$ in placebo group to $10.3 \%$ in tirofiban group $P=0.160){ }^{27}$ 


\section{Safety and tolerability of tirofiban}

The most serious and frequent adverse effects related to the use of GPIIb/IIIa receptor antagonists are bleeding and thrombocytopenia. Risk of bleeding can be reduced by the use of low-dose adjunctive heparin, early sheath removal, and meticulous post-procedure care. In studies involving tirofiban, the incidence of bleeding complications was more frequent than heparin alone, but major bleeding complications were not significantly different. Most of the increases in bleeding occurred in patients who underwent diagnostic catheterization or PCI, primarily at the femoral artery access site. The incidence of thrombocytopenia, defined as an absolute platelet count of less than $90,000 / \mathrm{mm}^{3}$, was $0.4 \%$ in PRISM and PRISM-PLUS trials. ${ }^{25,26}$ Finally in the Single High Dose Bolus Tirofiban and Sirolimus Eluting Stent in Myocardial Infarction (STRATEGY) trial, the use of tirofiban was associated with a trend towards reduction of any bleeding and to a statistically significant reduction in the incidence of thrombocytopenia $(P=0.03)$ compared with abciximab..$^{28}$

\section{Single high dose bolus tirofiban}

Tirofiban administration was shown to significantly reduce mortality, the composite of death or MI along with major adverse cardiovascular events (MACE) rate when compared with placebo. ${ }^{25-27}$ This benefit in ischemic endpoints reduction remained significant and of consistent magnitude in studies where tirofiban was tested in addition to thienopyridines but came at an increase risk for minor bleeding and thrombocytopenia. ${ }^{29} \mathrm{An}$ early ischemic hazard disfavouring tirofiban was noted when compared with abciximab in studies based on $10 \mathrm{mg} / \mathrm{kg}$ bolus regimen. ${ }^{30}$ Indeed, in the TARGET study tirofiban (RESTORE regimen, $10 \mu \mathrm{g} / \mathrm{kg}$ bolus $+0.15 \mu \mathrm{g} / \mathrm{kg} / \mathrm{min}$ infusion) was tested vs abciximab in moderate-high risk patients undergoing PCI and the primary endpoint (composite of death, nonfatal MI, and urgent target vessel revascularization within 30 days after procedure) occurred more frequently in the tirofiban group than in the abciximab group $(7.6 \%$ vs $6 \%, P=0.03) .{ }^{30}$ The superiority of abciximab was entirely driven by a higher rate of peri-procedural MI in the tirofiban arm. Tirofiban administrated immediately before PCI at RESTORE regimen was not sufficient and, as compared with abciximab, provided an inferior degree of platelet inhibition. As a consequence of the suboptimal platelet inhibition achieved, subsequent dose-ranging studies have led to an increase in the tirofiban bolus dose from 10 to $25 \mu \mathrm{g} / \mathrm{kg}$. The new single high dose bolus (SHDB) of tirofiban achieves an average inhibition of platelet aggregation of more than $90 \%$ throughout the first hour after treatment, which was shown to be comparable with that of abciximab. Subsequently, many studies have confirmed the safety and efficacy of SHDB tirofiban (eg, ADVANCE, ${ }^{31}$ STRATEGY ${ }^{28}$ MULTISTRATEGY, ${ }^{32}$ FATA $^{33}$ trials). The ADVANCE study included patients undergoing high-risk PCI, being or patients with diabetes and/or ACS or subjected to planned multivessel PCI. All patients were at steady-state for aspirin and thienopyridine and were randomized before PCI to SHDB tirofiban vs placebo. Tirofiban administration was able to reduce significantly myocardial infarction rate and GPIIb/IIIa bailout use. ${ }^{31}$ As a contrary, the STRATEGY and MULTISTRATEGY studies included patients with ST-segment elevation myocardial infarction. One of the aims of these studies was the comparison between SHDB tirofiban and abciximab, the GPIIb/IIIa inhibitor gold standard during primary PCI. No significant differences were found between tirofiban and abciximab (surrogate endpoint: ST-segment resolution $\geq 50 \%$ after primary $\mathrm{PCI}) .{ }^{28,32}$

\section{Tirofiban and daily clinical practice}

In conclusion, when used in high-risk patients, adjunctive tirofiban therapy, compared with placebo, is associated with a $\approx 30 \%$ reduction in all considered ischemic endpoints including overall mortality, mortality or MI, and MACE rates within 30 days after treatment. Importantly, the benefit observed soon after intervention persisted at the longest available follow-up. As expected, the advantage in terms of ischemic endpoints was counterbalanced by a significant increase in minor, but not major, bleeding and thrombocytopenia. ${ }^{29}$ When employed just prior to PCI, an early ischemic hazard disfavoring tirofiban was noted as compared with abciximab in studies based on $10 \mathrm{mg} / \mathrm{kg}$ bolus, ${ }^{27}$ whereas tirofiban at SHDB regimen may provide similar efficacy yet an improved safety profile. ${ }^{29}$

\section{Use of GPIIb/IIla inhibitors to overcome poor response to OAA during PCI}

The data indicating that low response to clopidogrel carries clinical significance are stronger than the data for aspirin. Nevertheless, several studies have clearly reported that aspirin and clopidogrel poor responders are at higher risk of peri-procedural MI, ${ }^{12,19}$ and that it is a known independent predictor of worse long-term clinical outcome. It is important to note that the risk carried by aspirin and/or clopidogrel poor response is higher and more frequent in high-risk patients, but it is present and significant also in low risk patients (eg, PCI for 
stable angina or silent ischemia or low-risk ACS). Nowadays, clinical approaches to overcome low response to aspirin or clopidogrel have not been established or examined systematically with randomized clinical trials. An initial approach would be to correct clinical factors that may contribute to low response or to decrease drug-drug interactions (eg, between aspirin and non-steroidal anti-inflammatory drugs or clopidogrel and proton pump inhibitors).

Another interesting strategy could be the tailored use of GPIIb/IIIa inhibitors in poor responder patients during PCI. This approach has been tested in two recent studies. Firstly, Cuisset et al have investigated the benefit of adjusted antiplatelet therapy with GPIIb/IIIa inhibitors (abciximab) in clopidogrel poor responders (using LTA to assess the drug response) ${ }^{34}$ In this study, a patient was considered poor responder whether platelet aggregation during aspirin and clopidogrel therapy at steady-state was $\geq 70 \% .149$ clopidogrel poor responders referred for elective PCI were included and randomized to 'conventional group' or 'active group' with GPIIb/IIIa inhibitors. The rate of cardiovascular events at 1 month was significantly lower in the 'active group' than in the 'conventional group' (19\% vs $40 \%$ respectively, $P=0.006) .{ }^{34}$ As a contrast, in the Tailoring Treatment With Tirofiban in Patients Showing Resistance to Aspirin and or Resistance to Clopidogrel (3T2R) study Valgimigli et al had screened 1277 patients to enrol 93 aspirin, 147 clopidogrel, and 23 dual poor responders (totally 263 poor responder patients randomized), who underwent elective PCI for stable or lowrisk unstable coronary artery disease. ${ }^{35}$ According to current guidelines, this subset of patients should be treated only with aspirin, clopidogrel, and unfractioned heparin bolus during the PCI procedure. The VerifyNow System was used to evaluate both aspirin and clopidogrel response. A patient was defined aspirin poor responder when ARU value was $\geq 550$; whereas a patient was considered clopidogrel poor responder when platelet inhibition by clopidogrel was $<40 \%$. Poor responders were randomly assigned in a double-blind manner to receive either SHDB tirofiban or placebo on top of standard aspirin and clopidogrel therapy. The primary endpoint was the rate of peri-procedural MI defined as an elevation of troponin $\mathrm{I} / \mathrm{T}$ ratio $\geq 3$ times the upper limit of normal (ULN) within 48 hours after completion of the PCI according to the recent universal definition of MI. The study showed that triple antiplatelet therapy, including a tailored infusion of tirofiban, in patients who responded poorly to aspirin, clopidogrel, or both, resulted in a $\approx 40 \%$ reduction in the incidence of periprocedural MI compared with standard care. ${ }^{35}$ The early benefit, in terms of peri-procedural MI, was largely maintained at 30 days, with the cumulative incidence of major adverse cardiovascular events reduced in the tirofiban group (Figure 2). Of note, no increase

$\square$ Placebo $\square$ Tirofiban

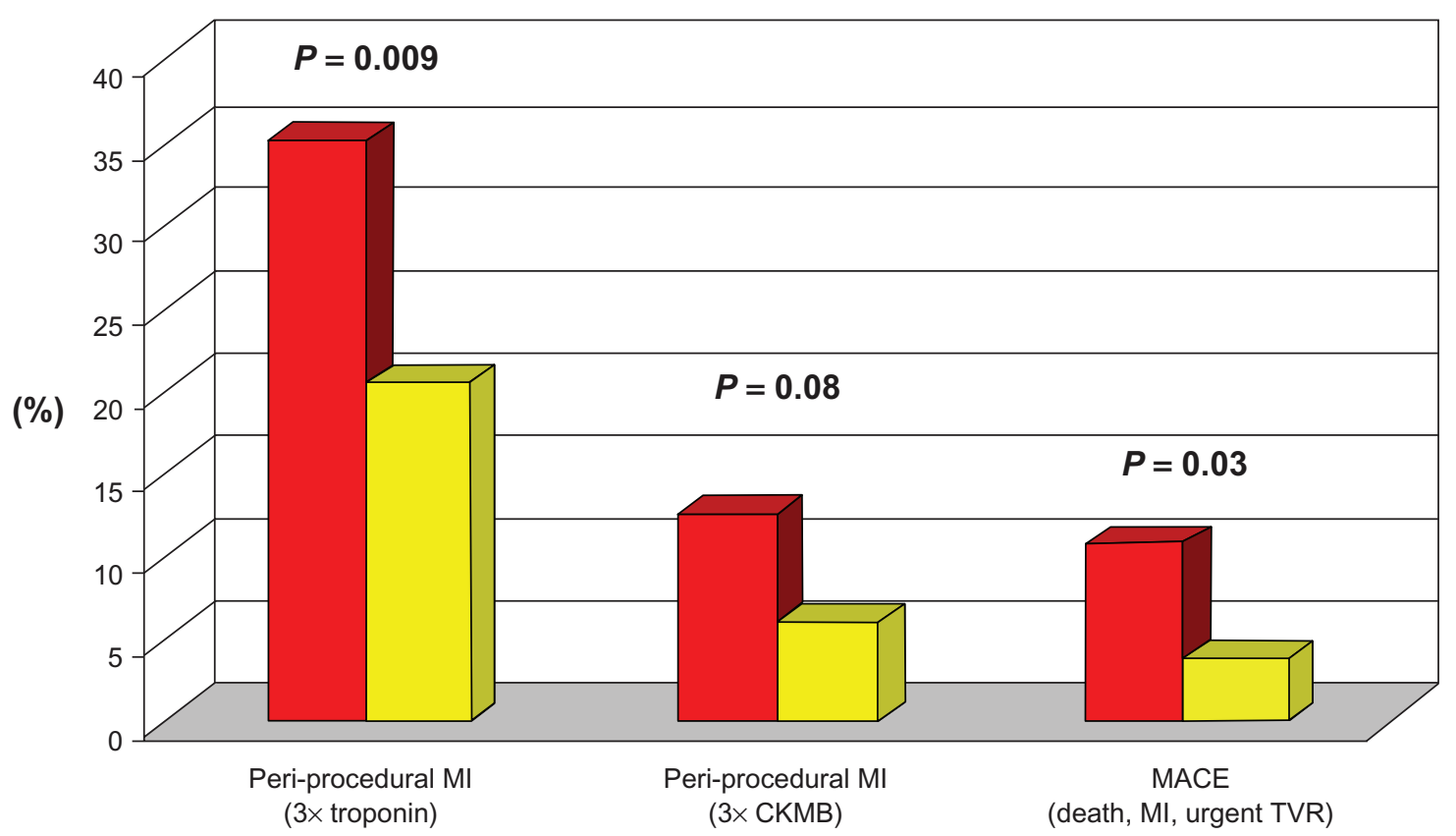

Figure 2 Principal endpoints in 3T/2R study.

Abbreviations: MI, myocardial infarction; MACE, major adverse cardiovascular events; $\mathrm{MI}$ in the mace count was defined as creatin-kinase MB (CKMB) $3 \times$ elevation upper normal limit;TVR, target vessel revascularization. 
in the risk of bleeding events was observed in the group of patients receiving tirofiban. ${ }^{35}$

\section{Conclusion}

Dual-antiplatelet therapy with aspirin and clopidogrel significantly reduces atherothrombotic events and improves long-term clinical outcomes in patients undergoing PCI and/or with ACS. However, despite dual-antiplatelet therapy, some patients still develop recurrent cardiovascular ischemic events. Many studies have clearly showed that a marked variability exists in the responsiveness to aspirin and clopidogrel, being the poor responder patients at higher risk of short- (peri-procedural) and long-term ischemic complications. Ongoing studies and new available antiplatelet drugs may permit to optimize their management and to improve their clinical outcome. Accordingly, the 3T/2R study showed that a tailored use of SHDB tirofiban in patients undergoing PCI, clinically at lowrisk being stable patients, but biologically at high-risk being aspirin and/or clopidogrel poor responders, is safe and effective reducing significantly the incidence of peri-procedural MI.

\section{Acknowledgments}

The authors thank Dr Monia Monti, Dr Stefania Gambetti, and Dr Laura Bristot (Medical Trials Analysis, Italy) for their assistance in collecting data.

\section{Disclosures}

The authors report no conflicts of interest in this work.

\section{References}

1. Antithrombotic Trialists' Collaboration. Collaborative meta-analysis of randomised trials of antiplatelet therapy for prevention of death, myocardial infarction, and stroke in high risk patients. BMJ. 2002;324: $71-86$.

2. CAPRIE Steering Committee. A randomised, blinded, trial of clopidogrel versus aspirin in patients at risk of ischaemic events (CAPRIE). Lancet. 1996;348:1329-1339.

3. Mehta SR, Yusuf S; for CURE Study Investigators. The Clopidogrel in Unstable angina to prevent Recurrent Events (CURE) trial programme; rationale, design and baseline characteristics including a meta-analysis of the effects of thienopyridines in vascular disease. Eur Heart $J$. 2000;21:2033-2041.

4. Mehta SR, Yusuf S. Peters RJ, et al. Effects of pretreatment with clopidogrel and aspirin followed by long-term therapy in patients undergoing percutaneous coronary intervention: the PCI-CURE study. Lancet. 2001;358:527-533.

5. Steinhubl SR, Berger PB, Tift Mann III J, et al. Early and sustained dual antiplatelet therapy following percutaneous coronary intervention. JAMA. 2002;288:2411-2420.

6. Angiolillo DJ, Fernandez-Ortiz A, Bernardo E, et al. Variability in individual responsiveness to clopidogrel: clinical implications, management, and future perspectives. J Am Coll Cardiol. 2007;49:1505-1516.

7.Simon T, Verstuyft C, Mary-Krause M, et al. Genetic determinants of response to clopidogrel and cardiovascular events. $N$ Engl $J$ Med. 2009;360(4):363-375.
8. Mega JL, Close DL, Wiviott SD, et al. Cytochrome P-450 polymorphisms and response to clopidogrel. N Engl J Med. 2009;360:354-362.

9. Gurbel PA, Bliden KP, Samara W, et al. Clopidogrel Effect on Platelet REactivity in Patients With Stent Thrombosis Results of the CREST Study. J Am Coll Cardiol. 2005;46:1827-1832.

10. Hochholzer W, Trenk D, Bestehorn HP, et al. Impact of the degree of peri-interventional platelet inhibition after loading with clopidogrel on early clinical outcome of elective coronary stent placement. $J$ Am Coll Cardiol. 2006;48:1742-1750.

11. Marcucci R, Gori AM, Paniccia R, et al. Cardiovascular death and nonfatal myocardial infarction in acute coronary syndrome patients receiving coronary stenting are predicted by residual platelet reactivity to ADP detected by a point-of-care assay: a 12-month follow-up. Circulation. 2009;119:237-242.

12. Patti G, Nusca A, Mangiacapra F, et al. Point-of-care measurement of clopidogrel responsiveness predicts clinical outcome in patients undergoing percutaneous coronary intervention results of the ARMYDA-PRO (Antiplatelet therapy for Reduction of MYocardial Damage during Angioplasty-Platelet Reactivity Predicts Outcome) study. J Am Coll Cardiol. 2008;52(14):1128-1133.

13. Sibbing D, Braun S, Morath T, et al. Platelet reactivity after clopidogrel treatment assessed with point-of-care analysis and early drug-eluting stent thrombosis. J Am Coll Cardiol. 2009;53:849-856.

14. Cattaneo M. Laboratory detection of 'aspirin resistance': what test should we use (if any)? Eur Heart J. 2007;28:1673-1675.

15. Campbell CL, Steinhubl SR. Variability in response to aspirin: do we understand the clinical relevance? J Thromb Haemost, 2005;3: 665-669.

16. Frelinger AL III, Furman MI, Linden MD, et al. Residual arachidonic acidinduced platelet activation via an adenosine diphosphate-dependent but cyclooxygenase-1- and cyclooxygenase-2-independent pathway: a 700patient study of aspirin resistance. Circulation. 2006;113:2888-2896.

17. Eikelboom JW, Hirsh J, Weitz JI, et al. Aspirin resistant thromboxane biosynthesis and the risk of myocardial infarction, stroke, or cardiovascular death in patients at high risk for cardiovascular events. Circulation. 2002;105:1650-1655.

18. Gum PA, Marchant K, et al. A prospective, blinded determination of the natural history of aspirin resistance among stable patients with cardiovascular disease. J Am Coll Cardiol. 2003;41:961-965.

19. Chen WH, Lee PY, Ng W, et al. Aspirin resistance is associated with a high incidence of myonecrosis after non-urgent percutaneous coronary intervention despite clopidogrel pretreatment. J Am Coll Cardiol. 2004;43:1122-1126.

20. O'Donoghue M, Braunwald E, Antman EA, et al. Pharmacodynamic effect and clinical efficacy of clopidogrel and prasugrel with or without a proton-pump inhibitor: an analysis of two randomised trials. Lancet. 2009;374:989-997.

21. Roffi M, Chew DP, Mukherjee D, et al. Platelet glicoprotein Iib/IIIa inhibitors reduce mortality in diabetic patients with non-ST segment elevation acute coronary syndromes. Circulation. 2004;104:2767-2771.

22. Campo G, Valgimigli M, Gemmati D, et al. Value of platelet reactivity in predicting response to treatment and clinical outcome in patients undergoing primary coronary intervention: insights into the STRATEGY study. J Am Coll Cardiol. 2006;48:2178-2185.

23. Cattaneo M. Aspirin and clopidogrel. Efficacy, safety and issue of drug resistance. Arterioscler Thromb Vasc Biol. 2004;24:1980-1987.

24. Schror K, Weber AA. Comparative pharmacology of GPIIb/IIIa antagonists. J Thrombosis Thrombolysis. 2003;15:71-80.

25. The PRISM study Investigators. A comparison of aspirin plus tirofiban with aspirin plus heparin for instable angina. NEJM. 1998;338:1498-1505.

26. The PRISM PLUS Investigators. Inhibition of the platelet glycoprotein IIb/IIIa receptor with tirofiban in unstable angina and non-Q-wave myocardial infarction. N Engl J Med. 1998;338:1488-1497.

27. The RESTORE Investigators. Effects of platelet glycoprotein IIb/IIIa blockade with torofiban on adverse cardiac events in patients with unstable angina or acute myocardial infarction undergoing coronary angioplasty. Circulation. 1997;96:1445-1453. 
28. Valgimigli M, Percoco G, Malagutti P, et al; STRATEGY Investigators. Tirofiban and sirolimus-eluting stent vs abciximab and bare-metal stent for acute myocardial infarction: a randomized trial. JAMA. 2005;293:2109-2117.

29. Valgimigli M, Biondi-Zoccai G, Tebaldi M, et al. Tirofiban as adjunctive therapy for acute coronary syndromes and percutaneous coronary intervention: a meta-analysis of randomized trials. Eur Heart J. 2009 Sep 14. [Epub ahead of print].

30. Stone GW, Moliterno DJ, Bertrand M, et al. Impact of clinical syndrome acuity on the differential responce to 2 glycoprotein IIb/IIIa inhibitors in patients undergoing coronary stenting the TARGET trial. Circulation. 2002;105:2347-2354.

31. Valgimigli M, Percoco G, Barbieri D, et al. The additive value of tirofiban administered with the high-dose bolus in the prevention of ischemic complications during high-risk coronary angioplasty: the ADVANCE Trial. J Am Coll Cardiol. 2004;44:14-19.

32. Valgimigli M, Campo G, Percoco G, et al. Comparison of angio- plasty with infusion of tirofiban or abciximab and with implantation of sirolimus-eluting or uncoated stents for acute myocardial infarction: the MULTI- STRATEGY randomized trial. JAMA. 2008;299:1788-1799.
33. Marzocchi A, Manari A, Piovaccari G, et al. Randomized comparison between tirofiban and abciximab to promote complete ST-resolution in primary angioplasty: results of the facilitated angioplasty with tirofiban or abciximab (FATA) in ST-elevation myocardial infarction trial. Eur Heart J. 2008;29:2972-2980.

34. Cuisset T, Frere C, Quilici J, et al. Glycoprotein IIb/IIIa inhibitors improve outcome after coronary stenting in clopidogrel nonresponders: a prospective, randomized study. JACC Cardiovasc Interv. 2008;1:649653.

35. Valgimigli M, Campo G, De Cesare N, et al. Intensifying platelet inhibition with tirofiban in poor responders to aspirin, clopidogrel, or both agents undergoing elective coronary intervention: results from the double-blind, prospective, randomized Tailoring Treatment with Tirofiban in Patients Showing Resistance to Aspirin and/or Resistance to Clopidogrel study. Circulation. 2009;119:3215-3222.
Journal of Blood Medicine

\section{Publish your work in this journal}

The Journal of Blood Medicine is an international, peer-reviewed, open access, online journal publishing laboratory, experimental and clinical aspects of all topics pertaining to blood based medicine including but not limited to: Transfusion Medicine; Blood collection, Donor issues, Transmittable diseases, and Blood banking logistics; Immunohematology; Artificial and alternative

\section{Dovepress}

blood based therapeutics; Hematology; Biotechnology/nanotechnology of blood related medicine; Legal aspects of blood medicine; Historical perspectives. The manuscript management system is completely online and includes a very quick and fair peer-review system. Visit http://www.dovepress.com/ testimonials.php to read real quotes from published authors.

Submit your manuscript here: http://www.dovepress.com/Journal-of-blood-medicine-journal 